\title{
Resistance mechanisms to third-generation epidermal growth factor receptor (EGFR) tyrosine kinase inhibitors in lung adenocarcinoma cells harboring the EGFR-T790M mutation
}

\author{
Kaori Nakatani ${ }^{1}$, Toshimitsu Yamaoka ${ }^{2}$, Tohru Ohmori ${ }^{2}$, Motoi Ohba ${ }^{2}$, Ken-Ichi Fujita ${ }^{2}$, lori Taki ${ }^{1}$, \\ Daisuke Kamei ${ }^{1}$, Shinichi lawai ${ }^{1}$, Yasutsuna Sasaki ${ }^{2}$ \\ ${ }^{I}$ Department of Health Care and Regulatory Sciences, Showa University School of Pharmacy, Japan, ${ }^{2}$ Institute of \\ Molecular Oncology, Showa University, Japan
}

Background: First- and second-generation EGFR tyrosine kinase inhibitors (TKIs) (e.g., gefitinib, erlotinib, and afatinib) are effective in patients with non-small cell lung cancer (NSCLC) harboring the EGFR-activating mutation. However, tumors develop resistance inevitably. Several resistance mechanisms have been proposed including emergence of the T790M mutation in EGFR exon 20, transformation to small cell lung cancer, and bypassing signaling pathways through Met, HER2, IGF1R, or Axl. The secondary mutation, EGFR-T790M, is the most common mechanism, and is found in $50 \%$ of resistant cases. Third-generation EGFR-TKIs, such as osimertinib and rociletinib, were developed to overcome resistance due to EGFR-T790M. However, acquired resistance still occurs. Therefore, clarifying acquired resistance mechanisms to third-generation EGFR-TKIs should be beneficial for patients with NSCLC.

Methods: PC-9 cells harbor the EGFR-activating mutation; a 15 bp deletion in exon 19. PC-9 cells were exposed to increasing concentrations of afatinib (maximum $1 \mu \mathrm{M}$ ) for 9-12 months to obtain afatinib-resistant (AfaR) cells. The EGFR-T790M mutation was detected in AfaR cells that were sensitive to osimertinib and rociletinib. Therefore, AfaR cells were exposed to increasing concentrations of osimertinib and rociletinib (maximum $1 \mu \mathrm{M}$ ) for 9-12 months individually to obtain cell lines resistant to osimertinib (OsiR1 and OsiR2) and rociletinib (RocR1 and RocR2). Cell proliferation was measured with the MTT assay and the expression of signal transduction proteins was determined by western blotting.

Results: AfaR cell proliferation was inhibited by osimertinib and rociletinib. OsiR1 and OsiR2 cells showed resistance to afatinib and rociletinib. However, RocR1 and RocR2 cells exhibited partial inhibition of proliferation by afatinib and osimertinib. Osimertinib and rociletinib suppressed EGFR phosphorylation in AfaR cells in a concentration-dependent matter, leading to inhibition of AKT and ERK1/2 activation. Furthermore, apoptosis was observed. EGFRphosphorylation, and the activation of AKT and ERK1/2, were not inhibited by osimertinib in OsiR1 and OsiR2 cells. EGFR-phosphorylation and AKT and ERK1/2 activation were slightly suppressed by rociletinib in RocR1 and RocR2 cells. These results suggest that there are different resistance mechanisms to osimertinib and rociletinib in NSCLC cells with the EGFR-T790M mutation.

Conclusion: Further studies on resistance mechanisms are important in understanding new therapeutic strategies for NSCLC patients. 